\title{
Pericardial Effusion and Tamponade: Making the Diagnosis at Bedside With Point-of-Care Echocardiography
}

Terry Singhapricha, MD; Todd Taylor, MD; Jehangir Meer, MD

\section{The authors demonstrate the utility of employing point-of-care echocardiography to detect pericardial effusion.}

\section{Background}

Pericardial effusions are usually an incidental finding on bedside echocardiogram-unless a patient shows clinical signs of tamponade physiology and obstructive shock. Echocardiogram is both sensitive and specific to detecting pericardial effusions. ${ }^{1}$ Although there are many causes of pericardial effusions, the most common causes of symptomatic effusions in the Western World are due to neoplasm, pericarditis, traumatic pathology, or idiopathic etiology. In developing countries, however, pericardial effusions are predominantly due to tuberculosis in an area where it is endemic. ${ }^{2,3}$ The size of the effusion is classified based on measurements of a fluid pocket during diastole. Mild effusion is defined as less than $10 \mathrm{~mm}$; moderate effusion, 10 to $20 \mathrm{~mm}$; and large effusion, greater than $20 \mathrm{~mm}^{3}$

It is important to note that the pericardial space contains up to $50 \mathrm{~mL}$ of physiological fluid which may be seen on echocardiography during systole. Small effusions usually contain less than $100 \mathrm{~mL}$ of fluid; moderate effusions contain 100 to $500 \mathrm{~mL}$ of fluid; and large effusions, over $500 \mathrm{~mL}$ of fluid. Fluid of smaller effusions typically layer posteriorly, while the fluid of some moderate and most large effusions may be seen circumferentially.

\section{Clinical Signs and Symptoms}

Patients with large chronic effusions are often asymptomatic, and clinical symptoms usually correlate to the acuity of pericardial accumulation. Patients with symptomatic effusions present with dyspnea on exertion that is followed by orthopnea, chest pain, and sometimes dysphagia, hoarseness, or hiccups due to irritation of surrounding structures until they exhibit tamponade physiology leading to hypotension secondary to obstructive shock. The most recognized signs of tamponade physiology on bedside echocardiography are early diastolic collapse of the right atrium and right ventricle, as well as ventricular interdependence. ${ }^{4}$

\section{Imaging Technique}

Pericardial effusion and cardiac tamponade can be detected in any of the standard echocardiographic views, with fluid usually appearing as an anechoic stripe. The fluid will first appear in the dependent portion of the pericardial space, but may become circumferential as it grows (Figure 1). After first determining the presence of an effusion, the right atrium and right ventricle are then evaluated for signs of diastolic collapse (Figure 2). Diastolic collapse can sometimes be difficult to visualize. In such cases, the motion modulation mode (M-mode)

Dr Singhapricha is a senior associate, department of emergency medicine, Emory University School of Medicine, Atlanta, Georgia. Dr Taylor is an assistant professor and assistant residency director, department of emergency medicine, Emory University School of Medicine, Atlanta, Georgia. Dr Meer is an assistant professor and director of emergency ultrasound, department of emergency medicine, Emory University School of Medicine, Atlanta, Georgia.

Authors' Disclosure Statement: The authors report no actual or potential conflict of interest in relation to this article.

DOI: 10.12788/emed.2017.0066 




Figure 1. An echocardiographic image taken in the parasternal longaxis view demonstrates moderate pericardial effusion.

can assist in visualizing the presence of a diastolic collapse (Figure 3).

The other sign of pericardial effusion is ventricular interdependence (Figure 4), which occurs when an increase in volume of one ventricle causes a decreased volume in the other. This is best seen in an apical four-chamber view and is secondary to chamber constriction caused by the effusion. During inspiration, the negative intrathoracic pressure leads to increased filling in the right atrium and ventricle. Due to the constriction caused by the effusion, the right ventricle can only expand against the septum, causing accentuated bowing against the left ventricle. During expiration, the increased intrathoracic pressure and the effusion lead to decreased filling of the right ventricle, causing the reverse effect.

After evaluating for signs of tamponade on examination, the next step is to evaluate the inferior vena cava (IVC). A simple way to identify the IVC on echocardiography is to visualize the heart in the subxiphoid view, then rotate the probe so that the marker is pointing toward the patient's head. As demonstrated in Figure 5, the IVC should be seen entering the right atrium; if the patient is truly in obstructive shock, the IVC should be plethoric with minimal respiratory variation.

\section{Pericardiocentesis}

In the event of obstructive shock or pulseless electric activity with visualized or suspected tamponade, pericardiocentesis is considered standard of care. There are many approaches to performing a pericardiocentesis, including the classically taught blind subxiphoid approach, which is associated with high rates of mor-

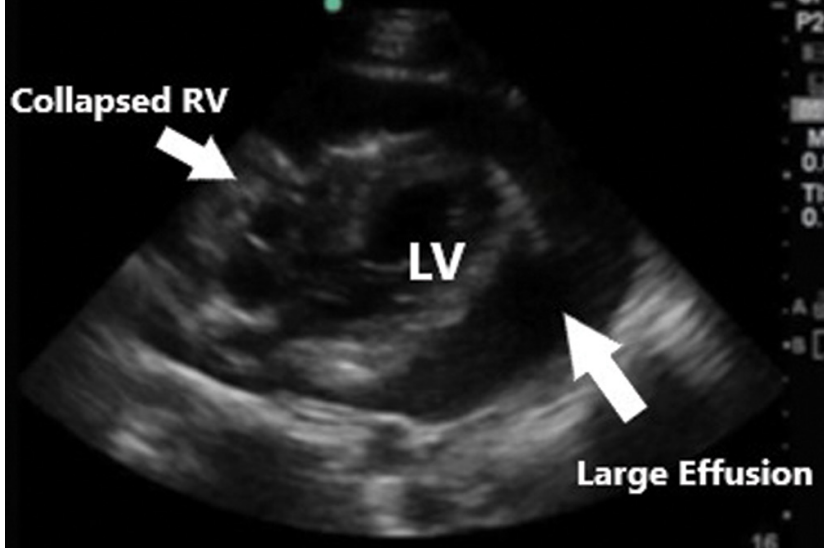

Figure 2. An echocardiographic image taken in the parasternal long-axis view demonstrates a large effusion causing right ventricular collapse during diastole.

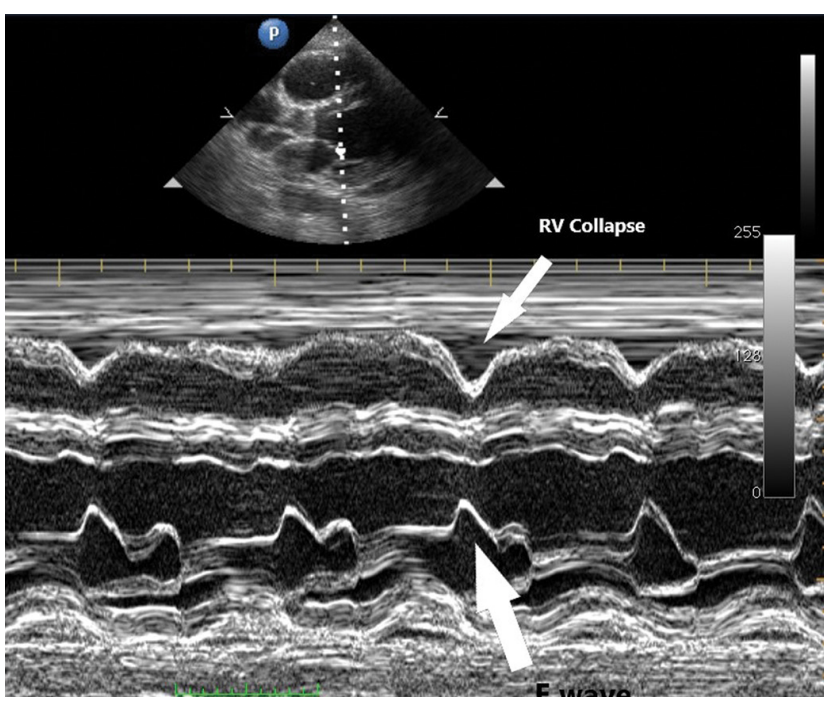

Figure 3. A motion modulation mode echocardiographic image in the parasternal long-axis view over the mitral valve demonstrates right ventricle diastolic collapse. The arrow indicates the mitral valve leaflet at its most open point.

bidity and mortality. ${ }^{5}$ More recent image-guided approaches employ echocardiography-guided techniques that identify the location and distribution of fluid, and perform pericardiocentesis closest to the area largest fluid accumulation.

Most of these guided techniques involve in-plane visualization of the needle in either a subxiphoid, apical, or parasternal approach. Studies have shown that the subxiphoid approach has a higher risk of injury to the liver, heart, and IVC, with complication rates up to $20 \%$ depending on the study. ${ }^{6}$ 


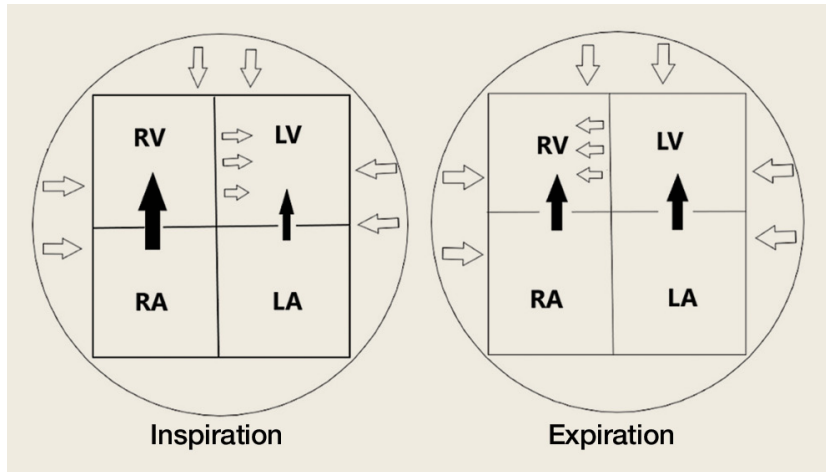

Figure 4. Illustration depicts ventricular interdependence with increased flow in the right ventricle during inspiration, causing septal bowing to the left and the reverse effect during expiration.

Abbreviations: LV, left ventricle; RV, right ventricle.

The apical approach involves locating the cardiac apex and inserting the needle $1 \mathrm{~cm}$ lateral to the apex, with the point directed toward the effusion and in-line with the ultrasound probe, taking care to avoid the lingula. Studies have shown that complication rates with this approach are around $3 \%{ }^{7}$

Recent studies also suggest that in-line medial-tolateral parasternal approaches may have minimal complications. However, when employing this approach, care must be taken to avoid the internal mammary artery, which can be identified using color-flow Doppler echocardiology. ${ }^{6}$

\section{Conclusion}

In general, bedside ultrasound is a quick and useful tool to evaluate for pericardial effusion and signs of tamponade physiology. When present, tamponade, a clinical diagnosis, is the likely cause of shock in the hemodynamically unstable patient with circumferential pericardial effusion.

While most cases of pericardial effusion are found incidentally, a stepwise approach to evaluate for tamponade is to quickly look for signs of early right-sided diastolic collapse or ventricular interdependence, as well

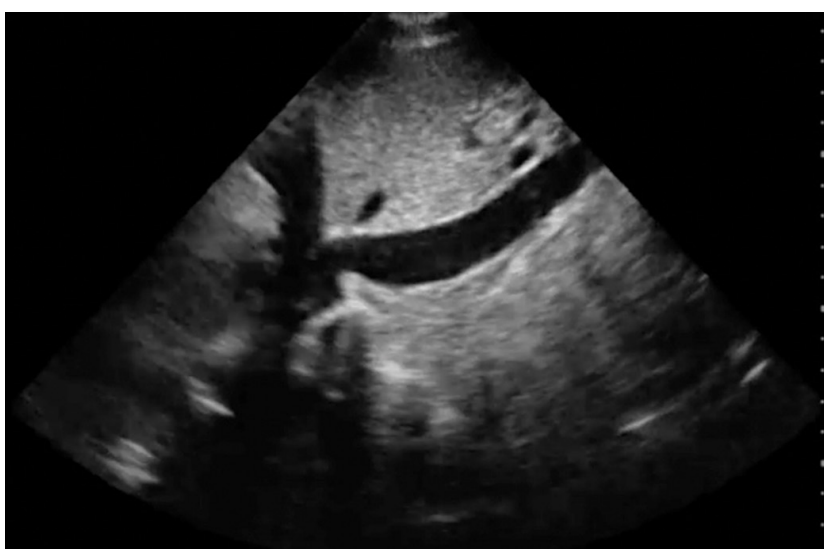

Figure 5. An echocardiographic image demonstrates a plethoric inferior vena cava with visible effusion.

as a plethoric IVC. For patients who have tamponade requiring pericardiocentesis, the ultrasound-guided apical or parasternal approaches have been shown to have fewer complications compared to the subxiphoid approach.

\section{References}

1. Imazio M, Adler Y. Management of pericardial effusion. Eur Heart J. 2013;34(16):1186-1197. doi:10.1093/eurheartj/ehs372.

2. Ben-Horin S, Bank I, Guetta V, Livneh A. Large symptomatic pericardial effusion as the presentation of unrecognized cancer: a study in 173 consecutive patients undergoing pericardiocentesis. Medicine. 2006;85(1):49-53. doi:10.1097/01.md.0000199556.69588.8e

3. Adler Y, Charron P, Imazio M, et al; European Society of Cardiology (ESC). 2015 ESC Guidelines for the diagnosis and management of pericardial diseases: The Task Force for the Diagnosis and Management of Pericardial Diseases of the European Society of Cardiology (ESC) Endorsed by: The European Association for Cardio-Thoracic Surgery (EACTS). Eur Heart J. 2015;36(42):2921-2964. doi:10.1093/ eurheartj/ehv318.

4. Nagdev A, Stone MB. Point-of-care ultrasound evaluation of pericardial effusions: does this patient have cardiac tamponade? Resuscitation. 2011;82(6):671-673. doi:10.1016/j.resuscitation.2011.02.004.

5. Kumar R, Sinha A, Lin MJ. Complications of pericardiocentesis: a clinical synopsis. Int J Crit Illn Inj Sci. 2015;5(3):206-212. doi:10.4103/2229-5151.165007.

6. Osman A, Wan Chuan T, Ab Rahman J, Via G, Tavazzi G. Ultrasoundguided pericardiocentesis: a novel parasternal approach. Eur J Emerg Med. 2017;5. doi:10.1097/MEJ.0000000000000471.

7. Ozer HO, Davutoğlu V, Cakici M. Echocardiography-guided pericardiocentesis with the apical approach. Turk Kardiyol Dern Ars. 2009;37(3):177-181. 